\title{
Stability of the 4-2 Binary Addition Circuit Cells. Part I
}

\author{
Katsumi Wasaki \\ Shinshu University \\ Nagano, Japan
}

\begin{abstract}
Summary. To evaluate our formal verification method on a real-size calculation circuit, in this article, we continue to formalize the concept of the 4-2 Binary Addition Cell primitives (FTAs) to define the structures of calculation units for a very fast multiplication algorithm for VLSI implementation [11]. We define the circuit structure of four-types FTAs, TYPE-0 to TYPE-3, using the series constructions of the Generalized Full Adder Circuits (GFAs) that generalized adder to have for each positive and negative weights to inputs and outputs [15]. We then successfully prove its circuit stability of the calculation outputs after four-steps. The motivation for this research is to establish a technique based on formalized mathematics and its applications for calculation circuits with high reliability.
\end{abstract}

MML identifier: FTACELL1, version: $\underline{7.9 .03 \quad 4.108 .1028}$

The terminology and notation used in this paper are introduced in the following papers: [8], [10], [14], [3], [13], [1], [7], [9], [6], [5], [4], [2], [12], and [15]. For simplicity the following abbreviations are introduced

$$
\begin{aligned}
\text { BitGFA } i \text { Str } & \longmapsto \Sigma_{i} \\
\text { BitGFA } i \text { Circ } & \longmapsto \mathfrak{C}_{i} \\
\text { GFA } i \text { AdderOutput } & \longmapsto \mathfrak{a}_{i} \\
\text { GFA } i \text { CarryOutput } & \longmapsto \mathfrak{c}_{i} \\
\text { InnerVertices } & \longmapsto \mathcal{I V}
\end{aligned}
$$




\section{Stability of 4-2 Binary Addition Circuit Cell (TyPe-0)}

Let $a_{1}, b_{1}, c_{1}, d_{1}, c_{2}$ be sets. The functor $\operatorname{BitFTA0Str}\left(a_{1}, b_{1}, c_{1}, d_{1}, c_{2}\right)$ yielding an unsplit non void strict non empty many sorted signature with arity held in gates and Boolean denotation held in gates is defined by:

(Def. 1) $\operatorname{BitFTA0Str}\left(a_{1}, b_{1}, c_{1}, d_{1}, c_{2}\right)=\Sigma_{0}\left(a_{1}, b_{1}, c_{1}\right)+\cdot \Sigma_{0}\left(\mathfrak{a}_{0}\left(a_{1}, b_{1}, c_{1}\right), c_{2}\right.$, $\left.d_{1}\right)$.

Let $a_{1}, b_{1}, c_{1}, d_{1}, c_{2}$ be sets. The functor $\operatorname{BitFTA0Circ}\left(a_{1}, b_{1}, c_{1}, d_{1}, c_{2}\right)$ yields a strict Boolean circuit of $\operatorname{BitFTA0Str}\left(a_{1}, b_{1}, c_{1}, d_{1}, c_{2}\right)$ with denotation held in gates and is defined as follows:

(Def. 2) $\operatorname{BitFTA0Circ~}\left(a_{1}, b_{1}, c_{1}, d_{1}, c_{2}\right)=\mathfrak{C}_{0}\left(a_{1}, b_{1}, c_{1}\right)+\cdot \mathfrak{C}_{0}\left(\mathfrak{a}_{0}\left(a_{1}, b_{1}, c_{1}\right), c_{2}\right.$, $\left.d_{1}\right)$.

One can prove the following propositions:

(1) Let $a_{1}, b_{1}, c_{1}, d_{1}, c_{2}$ be sets. Then $\mathcal{I V}\left(\operatorname{BitFTA} 0 \operatorname{Str}\left(a_{1}, b_{1}, c_{1}, d_{1}, c_{2}\right)\right)=$ $\left\{\left\langle\left\langle a_{1}, b_{1}\right\rangle, \operatorname{xor}_{2}\right\rangle, \mathfrak{a}_{0}\left(a_{1}, b_{1}, c_{1}\right)\right\} \cup\left\{\left\langle\left\langle a_{1}, b_{1}\right\rangle\right.\right.$, and $\left._{2}\right\rangle,\left\langle\left\langle b_{1}, c_{1}\right\rangle, \operatorname{and}_{2}\right\rangle,\left\langle\left\langle c_{1}\right.\right.$, $\left.\left.\left.a_{1}\right\rangle, \operatorname{and}_{2}\right\rangle, \mathfrak{c}_{0}\left(a_{1}, b_{1}, c_{1}\right)\right\} \cup\left\{\left\langle\left\langle\mathfrak{a}_{0}\left(a_{1}, b_{1}, c_{1}\right), c_{2}\right\rangle, \operatorname{xor}_{2}\right\rangle, \mathfrak{a}_{0}\left(\mathfrak{a}_{0}\left(a_{1}, b_{1}, c_{1}\right)\right.\right.$, $\left.\left.c_{2}, d_{1}\right)\right\} \cup\left\{\left\langle\left\langle\mathfrak{a}_{0}\left(a_{1}, b_{1}, c_{1}\right), c_{2}\right\rangle, \operatorname{and}_{2}\right\rangle,\left\langle\left\langle c_{2}, d_{1}\right\rangle, \operatorname{and}_{2}\right\rangle,\left\langle\left\langle d_{1}, \mathfrak{a}_{0}\left(a_{1}, b_{1}, c_{1}\right)\right\rangle\right.\right.$, $\left.\left.\operatorname{and}_{2}\right\rangle, \mathfrak{c}_{0}\left(\mathfrak{a}_{0}\left(a_{1}, b_{1}, c_{1}\right), c_{2}, d_{1}\right)\right\}$.

(2) For all sets $a_{1}, b_{1}, c_{1}, d_{1}, c_{2}$ holds $\mathcal{I V}\left(\operatorname{BitFTA0Str}\left(a_{1}, b_{1}, c_{1}, d_{1}, c_{2}\right)\right)$ is a binary relation.

(3) For all non pair sets $a_{1}, b_{1}, c_{1}, d_{1}$ and for every set $c_{2}$ such that $c_{2} \neq\left\langle\left\langle d_{1}, \mathfrak{a}_{0}\left(a_{1}, b_{1}, c_{1}\right)\right\rangle\right.$, and $\left.\operatorname{an}_{2}\right\rangle$ and $c_{2} \notin \mathcal{I V}\left(\Sigma_{0}\left(a_{1}, b_{1}, c_{1}\right)\right)$ holds InputVertices(BitFTA0Str $\left.\left(a_{1}, b_{1}, c_{1}, d_{1}, c_{2}\right)\right)=\left\{a_{1}, b_{1}, c_{1}, d_{1}, c_{2}\right\}$.

(4) Let $a_{1}, b_{1}, c_{1}, d_{1}, c_{2}$ be sets. Then $a_{1} \in$ the carrier of $\operatorname{BitFTA0Str}\left(a_{1}\right.$, $\left.b_{1}, c_{1}, d_{1}, c_{2}\right)$ and $b_{1} \in$ the carrier of $\operatorname{BitFTA0Str}\left(a_{1}, b_{1}, c_{1}, d_{1}, c_{2}\right)$ and $c_{1} \in$ the carrier of $\operatorname{BitFTA0Str}\left(a_{1}, b_{1}, c_{1}, d_{1}, c_{2}\right)$ and $d_{1} \in$ the carrier of $\operatorname{BitFTA0Str}\left(a_{1}, b_{1}, c_{1}, d_{1}, c_{2}\right)$ and $c_{2} \in$ the carrier of $\operatorname{BitFTA0Str}\left(a_{1}\right.$, $\left.b_{1}, c_{1}, d_{1}, c_{2}\right)$ and $\left\langle\left\langle a_{1}, b_{1}\right\rangle\right.$, xor $\left._{2}\right\rangle \in$ the carrier of $\operatorname{BitFTA0Str}\left(a_{1}, b_{1}, c_{1}\right.$, $\left.d_{1}, c_{2}\right)$ and $\mathfrak{a}_{0}\left(a_{1}, b_{1}, c_{1}\right) \in$ the carrier of $\operatorname{BitFTA0Str}\left(a_{1}, b_{1}, c_{1}, d_{1}, c_{2}\right)$ and $\left\langle\left\langle a_{1}, b_{1}\right\rangle\right.$, and $\left._{2}\right\rangle \in$ the carrier of $\operatorname{BitFTA} 0 \operatorname{Str}\left(a_{1}, b_{1}, c_{1}, d_{1}, c_{2}\right)$ and $\left\langle\left\langle b_{1}, c_{1}\right\rangle, \operatorname{and}_{2}\right\rangle \in$ the carrier of $\operatorname{BitFTA} 0 \operatorname{Str}\left(a_{1}, b_{1}, c_{1}, d_{1}, c_{2}\right)$ and $\left\langle\left\langle c_{1}\right.\right.$, $\left.\left.a_{1}\right\rangle, \operatorname{and}_{2}\right\rangle \in$ the carrier of $\operatorname{BitFTA} \operatorname{Str}\left(a_{1}, b_{1}, c_{1}, d_{1}, c_{2}\right)$ and $\mathfrak{c}_{0}\left(a_{1}, b_{1}\right.$, $\left.c_{1}\right) \in$ the carrier of $\operatorname{BitFTA0Str}\left(a_{1}, b_{1}, c_{1}, d_{1}, c_{2}\right)$ and $\left\langle\left\langle\mathfrak{a}_{0}\left(a_{1}, b_{1}, c_{1}\right), c_{2}\right\rangle\right.$, xor $\left._{2}\right\rangle \in$ the carrier of $\operatorname{BitFTA0Str}\left(a_{1}, b_{1}, c_{1}, d_{1}, c_{2}\right)$ and $\mathfrak{a}_{0}\left(\mathfrak{a}_{0}\left(a_{1}, b_{1}, c_{1}\right)\right.$, $\left.c_{2}, d_{1}\right) \in$ the carrier of $\operatorname{BitFTA0Str}\left(a_{1}, b_{1}, c_{1}, d_{1}, c_{2}\right)$ and $\left\langle\left\langle\mathfrak{a}_{0}\left(a_{1}, b_{1}, c_{1}\right)\right.\right.$, $\left.\left.c_{2}\right\rangle, \operatorname{and}_{2}\right\rangle \in$ the carrier of $\operatorname{BitFTA0Str}\left(a_{1}, b_{1}, c_{1}, d_{1}, c_{2}\right)$ and $\left\langle\left\langle c_{2}, d_{1}\right\rangle\right.$, $\left.\operatorname{and}_{2}\right\rangle \in$ the carrier of $\operatorname{BitFTA0Str}\left(a_{1}, b_{1}, c_{1}, d_{1}, c_{2}\right)$ and $\left\langle\left\langle d_{1}, \mathfrak{a}_{0}\left(a_{1}, b_{1}\right.\right.\right.$, $\left.\left.c_{1}\right)\right\rangle$, and $\left.\operatorname{an}_{2}\right\rangle \in$ the carrier of $\operatorname{BitFTA0Str}\left(a_{1}, b_{1}, c_{1}, d_{1}, c_{2}\right)$ and $\mathfrak{c}_{0}\left(\mathfrak{a}_{0}\left(a_{1}\right.\right.$, $\left.\left.b_{1}, c_{1}\right), c_{2}, d_{1}\right) \in$ the carrier of $\operatorname{BitFTA0Str}\left(a_{1}, b_{1}, c_{1}, d_{1}, c_{2}\right)$.

(5) Let $a_{1}, b_{1}, c_{1}, d_{1}, c_{2}$ be sets. Then $\left\langle\left\langle a_{1}, b_{1}\right\rangle, \operatorname{xor}_{2}\right\rangle \in \mathcal{I} \mathcal{V}\left(\operatorname{BitFTA} 0 \operatorname{Str}\left(a_{1}\right.\right.$, $\left.\left.b_{1}, c_{1}, d_{1}, c_{2}\right)\right)$ and $\mathfrak{a}_{0}\left(a_{1}, b_{1}, c_{1}\right) \in \mathcal{I} \mathcal{V}\left(\operatorname{BitFTA} 0 \operatorname{Str}\left(a_{1}, b_{1}, c_{1}, d_{1}, c_{2}\right)\right)$ and 
$\left\langle\left\langle a_{1}, b_{1}\right\rangle, \operatorname{and}_{2}\right\rangle,\left\langle\left\langle b_{1}, c_{1}\right\rangle, \operatorname{and}_{2}\right\rangle,\left\langle\left\langle c_{1}, a_{1}\right\rangle, \operatorname{and}_{2}\right\rangle \in \mathcal{I V}\left(\operatorname{BitFTA} 0 \operatorname{Str}\left(a_{1}\right.\right.$, $\left.\left.b_{1}, c_{1}, d_{1}, c_{2}\right)\right)$ and $\mathfrak{c}_{0}\left(a_{1}, b_{1}, c_{1}\right) \in \mathcal{I V}\left(\operatorname{BitFTA} 0 \operatorname{Str}\left(a_{1}, b_{1}, c_{1}, d_{1}, c_{2}\right)\right)$ and $\left\langle\left\langle\mathfrak{a}_{0}\left(a_{1}, b_{1}, c_{1}\right), c_{2}\right\rangle, \operatorname{xor}_{2}\right\rangle, \mathfrak{a}_{0}\left(\mathfrak{a}_{0}\left(a_{1}, b_{1}, c_{1}\right), c_{2}, d_{1}\right),\left\langle\left\langle\mathfrak{a}_{0}\left(a_{1}, b_{1}, c_{1}\right)\right.\right.$, $\left.\left.c_{2}\right\rangle, \operatorname{and}_{2}\right\rangle,\left\langle\left\langle c_{2}, d_{1}\right\rangle, \operatorname{and}_{2}\right\rangle,\left\langle\left\langle d_{1}, \mathfrak{a}_{0}\left(a_{1}, b_{1}, c_{1}\right)\right\rangle, \operatorname{and}_{2}\right\rangle, \mathfrak{c}_{0}\left(\mathfrak{a}_{0}\left(a_{1}, b_{1}, c_{1}\right)\right.$, $\left.c_{2}, d_{1}\right) \in \mathcal{I} \mathcal{V}\left(\operatorname{BitFTA} 0 \operatorname{Str}\left(a_{1}, b_{1}, c_{1}, d_{1}, c_{2}\right)\right)$.

(6) Let $a_{1}, b_{1}, c_{1}, d_{1}$ be non pair sets and $c_{2}$ be a set. Suppose $c_{2} \neq\left\langle\left\langle d_{1}\right.\right.$, $\left.\left.\mathfrak{a}_{0}\left(a_{1}, b_{1}, c_{1}\right)\right\rangle, \operatorname{and}_{2}\right\rangle$ and $c_{2} \notin \mathcal{I V}\left(\Sigma_{0}\left(a_{1}, b_{1}, c_{1}\right)\right)$. Then $a_{1}, b_{1}, c_{1}, d_{1}$,

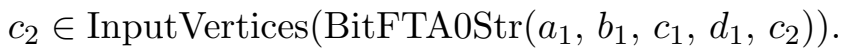

Let $a_{1}, b_{1}, c_{1}, d_{1}, c_{2}$ be sets. The functor BitFTA0CarryOutput $\left(a_{1}, b_{1}, c_{1}\right.$, $\left.d_{1}, c_{2}\right)$ yields an element of $\mathcal{I V}\left(\operatorname{BitFTA} 0 \operatorname{Str}\left(a_{1}, b_{1}, c_{1}, d_{1}, c_{2}\right)\right)$ and is defined as follows:

(Def. 3) BitFTA0CarryOutput $\left(a_{1}, b_{1}, c_{1}, d_{1}, c_{2}\right)=\mathfrak{c}_{0}\left(a_{1}, b_{1}, c_{1}\right)$.

The functor $\operatorname{BitFTA0AdderOutputI}\left(a_{1}, b_{1}, c_{1}, d_{1}, c_{2}\right)$ yields an element of $\mathcal{I V}$ (BitFTA0Str $\left.\left(a_{1}, b_{1}, c_{1}, d_{1}, c_{2}\right)\right)$ and is defined as follows:

(Def. 4) BitFTA0AdderOutputI $\left(a_{1}, b_{1}, c_{1}, d_{1}, c_{2}\right)=\mathfrak{a}_{0}\left(a_{1}, b_{1}, c_{1}\right)$.

The functor $\operatorname{BitFTA0AdderOutputP}\left(a_{1}, b_{1}, c_{1}, d_{1}, c_{2}\right)$ yielding an element of $\mathcal{I V}\left(\operatorname{BitFTA0Str}\left(a_{1}, b_{1}, c_{1}, d_{1}, c_{2}\right)\right)$ is defined by:

(Def. 5) BitFTA0AdderOutputP $\left(a_{1}, b_{1}, c_{1}, d_{1}, c_{2}\right)=\mathfrak{c}_{0}\left(\mathfrak{a}_{0}\left(a_{1}, b_{1}, c_{1}\right), c_{2}, d_{1}\right)$.

The functor BitFTA0AdderOutputQ $\left(a_{1}, b_{1}, c_{1}, d_{1}, c_{2}\right)$ yields an element of $\mathcal{I V}\left(\operatorname{BitFTA} \operatorname{Str}\left(a_{1}, b_{1}, c_{1}, d_{1}, c_{2}\right)\right)$ and is defined by:

(Def. 6) BitFTA0AdderOutputQ $\left(a_{1}, b_{1}, c_{1}, d_{1}, c_{2}\right)=\mathfrak{a}_{0}\left(\mathfrak{a}_{0}\left(a_{1}, b_{1}, c_{1}\right), c_{2}, d_{1}\right)$.

The following propositions are true:

(7) Let $a_{1}, b_{1}, c_{1}$ be non pair sets, $d_{1}, c_{2}$ be sets, $s$ be a state of $\operatorname{BitFTA0Circ}\left(a_{1}, b_{1}, c_{1}, d_{1}, c_{2}\right)$, and $a_{2}, a_{3}, a_{4}$ be elements of Boolean. Suppose $a_{2}=s\left(a_{1}\right)$ and $a_{3}=s\left(b_{1}\right)$ and $a_{4}=s\left(c_{1}\right)$. Then (Following $(s, 2))\left(\operatorname{BitFTA0CarryOutput}\left(a_{1}, b_{1}, c_{1}, d_{1}, c_{2}\right)\right)=a_{2} \wedge a_{3} \vee a_{3} \wedge$ $a_{4} \vee a_{4} \wedge a_{2}$ and (Following $\left.(s, 2)\right)$ (BitFTA0AdderOutputI $\left(a_{1}, b_{1}, c_{1}, d_{1}\right.$, $\left.\left.c_{2}\right)\right)=a_{2} \oplus a_{3} \oplus a_{4}$.

(8) Let $a_{1}, b_{1}, c_{1}, d_{1}$ be non pair sets and $c_{2}$ be a set. Suppose $c_{2} \neq\left\langle\left\langle d_{1}\right.\right.$, $\left.\mathfrak{a}_{0}\left(a_{1}, b_{1}, c_{1}\right)\right\rangle$, and $\left.{ }_{2}\right\rangle$ and $c_{2} \notin \mathcal{I V}\left(\Sigma_{0}\left(a_{1}, b_{1}, c_{1}\right)\right)$. Let $s$ be a state of $\operatorname{BitFTA0Circ}\left(a_{1}, b_{1}, c_{1}, d_{1}, c_{2}\right)$ and $a_{2}, a_{3}, a_{4}, a_{5}, a_{6}$ be elements of Boolean. Suppose $a_{2}=s\left(a_{1}\right)$ and $a_{3}=s\left(b_{1}\right)$ and $a_{4}=s\left(c_{1}\right)$ and $a_{5}=s\left(d_{1}\right)$ and $a_{6}=s\left(c_{2}\right)$. Then $($ Following $(s, 2))\left(\mathfrak{a}_{0}\left(a_{1}, b_{1}, c_{1}\right)\right)=$ $a_{2} \oplus a_{3} \oplus a_{4}$ and (Following $\left.(s, 2)\right)\left(a_{1}\right)=a_{2}$ and (Following $\left.(s, 2)\right)\left(b_{1}\right)=$ $a_{3}$ and (Following $\left.(s, 2)\right)\left(c_{1}\right)=a_{4}$ and $($ Following $(s, 2))\left(d_{1}\right)=a_{5}$ and (Following $(s, 2))\left(c_{2}\right)=a_{6}$.

(9) Let $a_{1}, b_{1}, c_{1}, d_{1}$ be non pair sets and $c_{2}$ be a set. Suppose $c_{2} \neq\left\langle\left\langle d_{1}\right.\right.$, $\left.\mathfrak{a}_{0}\left(a_{1}, b_{1}, c_{1}\right)\right\rangle$, $\left.\operatorname{and}_{2}\right\rangle$ and $c_{2} \notin \mathcal{I V}\left(\Sigma_{0}\left(a_{1}, b_{1}, c_{1}\right)\right)$. Let $s$ be a state of $\operatorname{BitFTA0Circ}\left(a_{1}, b_{1}, c_{1}, d_{1}, c_{2}\right)$ and $a_{2}, a_{3}, a_{4}, a_{5}, a_{6}$ be elements of Boolean. Suppose $a_{2}=s\left(a_{1}\right)$ and $a_{3}=s\left(b_{1}\right)$ and $a_{4}=s\left(c_{1}\right)$ and $a_{5}=s\left(d_{1}\right)$ 
and $a_{6}=s\left(c_{2}\right)$. Then (Following $\left.(s, 4)\right)$ (BitFTA0AdderOutputP $\left(a_{1}, b_{1}\right.$, $\left.\left.c_{1}, d_{1}, c_{2}\right)\right)=\left(a_{2} \oplus a_{3} \oplus a_{4}\right) \wedge a_{6} \vee a_{6} \wedge a_{5} \vee a_{5} \wedge\left(a_{2} \oplus a_{3} \oplus a_{4}\right)$ and (Following $(s, 4))\left(\operatorname{BitFTA0AdderOutputQ}\left(a_{1}, b_{1}, c_{1}, d_{1}, c_{2}\right)\right)=a_{2} \oplus a_{3} \oplus$ $a_{4} \oplus a_{5} \oplus a_{6}$.

(10) Let $a_{1}, b_{1}, c_{1}, d_{1}$ be non pair sets and $c_{2}$ be a set. If $c_{2} \neq\left\langle\left\langle d_{1}, \mathfrak{a}_{0}\left(a_{1}, b_{1}\right.\right.\right.$, $\left.\left.\left.c_{1}\right)\right\rangle, \operatorname{and}_{2}\right\rangle$, then for every state $s$ of $\operatorname{BitFTA0Circ}\left(a_{1}, b_{1}, c_{1}, d_{1}, c_{2}\right)$ holds Following $(s, 4)$ is stable.

\section{Stability of 4-2 Binary Addition Circuit Cell (TYPE-1)}

Let $a_{1}, b_{2}, c_{1}, d_{2}, c_{2}$ be sets. The functor $\operatorname{BitFTA} 1 \operatorname{Str}\left(a_{1}, b_{2}, c_{1}, d_{2}, c_{2}\right)$ yielding an unsplit non void strict non empty many sorted signature with arity held in gates and Boolean denotation held in gates is defined by:

(Def. 7) $\operatorname{BitFTA1Str}\left(a_{1}, b_{2}, c_{1}, d_{2}, c_{2}\right)=\Sigma_{1}\left(a_{1}, b_{2}, c_{1}\right)+\cdot \Sigma_{2}\left(\mathfrak{a}_{1}\left(a_{1}, b_{2}, c_{1}\right), c_{2}\right.$, $\left.d_{2}\right)$.

Let $a_{1}, b_{2}, c_{1}, d_{2}, c_{2}$ be sets. The functor $\operatorname{BitFTA1Circ}\left(a_{1}, b_{2}, c_{1}, d_{2}, c_{2}\right)$ yields a strict Boolean circuit of $\operatorname{BitFTA} 1 \operatorname{Str}\left(a_{1}, b_{2}, c_{1}, d_{2}, c_{2}\right)$ with denotation held in gates and is defined by:

(Def. 8) $\operatorname{BitFTA1Circ}\left(a_{1}, b_{2}, c_{1}, d_{2}, c_{2}\right)=\mathfrak{C}_{1}\left(a_{1}, b_{2}, c_{1}\right)+\cdot \mathfrak{C}_{2}\left(\mathfrak{a}_{1}\left(a_{1}, b_{2}, c_{1}\right), c_{2}\right.$, $\left.d_{2}\right)$.

Next we state several propositions:

(11) Let $a_{1}, b_{2}, c_{1}, d_{2}, c_{2}$ be sets. Then $\mathcal{I V}\left(\operatorname{BitFTA} 1 \operatorname{Str}\left(a_{1}, b_{2}, c_{1}, d_{2}\right.\right.$, $\left.\left.c_{2}\right)\right)=\left\{\left\langle\left\langle a_{1}, b_{2}\right\rangle\right.\right.$, xor2c $\left.\rangle, \mathfrak{a}_{1}\left(a_{1}, b_{2}, c_{1}\right)\right\} \cup\left\{\left\langle\left\langle a_{1}, b_{2}\right\rangle\right.\right.$, and2c $\rangle,\left\langle\left\langle b_{2}, c_{1}\right\rangle\right.$, $\left.\left.\operatorname{and}_{2 a}\right\rangle,\left\langle\left\langle c_{1}, a_{1}\right\rangle, \operatorname{and}_{2}\right\rangle, \mathfrak{c}_{1}\left(a_{1}, b_{2}, c_{1}\right)\right\} \cup\left\{\left\langle\left\langle\mathfrak{a}_{1}\left(a_{1}, b_{2}, c_{1}\right), c_{2}\right\rangle\right.\right.$, xor2c $\rangle, \mathfrak{a}_{2}\left(\mathfrak{a}_{1}\right.$ $\left.\left.\left(a_{1}, b_{2}, c_{1}\right), c_{2}, d_{2}\right)\right\} \cup\left\{\left\langle\left\langle\mathfrak{a}_{1}\left(a_{1}, b_{2}, c_{1}\right), c_{2}\right\rangle, \operatorname{and}_{2 a}\right\rangle,\left\langle\left\langle c_{2}, d_{2}\right\rangle\right.\right.$, and2c $\rangle,\left\langle\left\langle d_{2}\right.\right.$, $\left.\mathfrak{a}_{1}\left(a_{1}, b_{2}, c_{1}\right)\right\rangle$, and $\left.\left.\operatorname{and}_{2 b}\right\rangle, \mathfrak{c}_{2}\left(\mathfrak{a}_{1}\left(a_{1}, b_{2}, c_{1}\right), c_{2}, d_{2}\right)\right\}$.

(12) For all sets $a_{1}, b_{2}, c_{1}, d_{2}, c_{2}$ holds $\mathcal{I V}\left(\operatorname{BitFTA} 1 \operatorname{Str}\left(a_{1}, b_{2}, c_{1}, d_{2}, c_{2}\right)\right)$ is a binary relation.

(13) For all non pair sets $a_{1}, b_{2}, c_{1}, d_{2}$ and for every set $c_{2}$ such that $c_{2} \neq\left\langle\left\langle d_{2}, \mathfrak{a}_{1}\left(a_{1}, b_{2}, c_{1}\right)\right\rangle\right.$, and $\left.{ }_{2 b}\right\rangle$ and $c_{2} \notin \mathcal{I V}\left(\Sigma_{1}\left(a_{1}, b_{2}, c_{1}\right)\right)$ holds InputVertices(BitFTA1Str $\left.\left(a_{1}, b_{2}, c_{1}, d_{2}, c_{2}\right)\right)=\left\{a_{1}, b_{2}, c_{1}, d_{2}, c_{2}\right\}$.

(14) Let $a_{1}, b_{2}, c_{1}, d_{2}, c_{2}$ be sets. Then $a_{1} \in$ the carrier of $\operatorname{BitFTA} \operatorname{Str}\left(a_{1}\right.$, $\left.b_{2}, c_{1}, d_{2}, c_{2}\right)$ and $b_{2} \in$ the carrier of $\operatorname{BitFTA} 1 \operatorname{Str}\left(a_{1}, b_{2}, c_{1}, d_{2}, c_{2}\right)$ and $c_{1} \in$ the carrier of $\operatorname{BitFTA} 1 \operatorname{Str}\left(a_{1}, b_{2}, c_{1}, d_{2}, c_{2}\right)$ and $d_{2} \in$ the carrier of $\operatorname{BitFTA} 1 \operatorname{Str}\left(a_{1}, b_{2}, c_{1}, d_{2}, c_{2}\right)$ and $c_{2} \in$ the carrier of $\operatorname{BitFTA} 1 \operatorname{Str}\left(a_{1}, b_{2}\right.$, $\left.c_{1}, d_{2}, c_{2}\right)$ and $\left\langle\left\langle a_{1}, b_{2}\right\rangle\right.$, xor2c $\rangle \in$ the carrier of $\operatorname{BitFTA} 1 \operatorname{Str}\left(a_{1}, b_{2}, c_{1}\right.$, $\left.d_{2}, c_{2}\right)$ and $\mathfrak{a}_{1}\left(a_{1}, b_{2}, c_{1}\right) \in$ the carrier of $\operatorname{BitFTA} 1 \operatorname{Str}\left(a_{1}, b_{2}, c_{1}, d_{2}, c_{2}\right)$ and $\left\langle\left\langle a_{1}, b_{2}\right\rangle\right.$, and2c $\rangle \in$ the carrier of $\operatorname{BitFTA} 1 \operatorname{Str}\left(a_{1}, b_{2}, c_{1}, d_{2}, c_{2}\right)$ and $\left\langle\left\langle b_{2}, c_{1}\right\rangle, \operatorname{and}_{2 a}\right\rangle \in$ the carrier of $\operatorname{BitFTA} 1 \operatorname{Str}\left(a_{1}, b_{2}, c_{1}, d_{2}, c_{2}\right)$ and $\left\langle\left\langle c_{1}\right.\right.$, $\left.\left.a_{1}\right\rangle, \operatorname{and}_{2}\right\rangle \in$ the carrier of $\operatorname{BitFTA} 1 \operatorname{Str}\left(a_{1}, b_{2}, c_{1}, d_{2}, c_{2}\right)$ and $\mathfrak{c}_{1}\left(a_{1}, b_{2}\right.$, 
$\left.c_{1}\right) \in$ the carrier of $\operatorname{BitFTA} 1 \operatorname{Str}\left(a_{1}, b_{2}, c_{1}, d_{2}, c_{2}\right)$ and $\left\langle\left\langle\mathfrak{a}_{1}\left(a_{1}, b_{2}, c_{1}\right), c_{2}\right\rangle\right.$, xor2c $\rangle \in$ the carrier of $\operatorname{BitFTA} 1 \operatorname{Str}\left(a_{1}, b_{2}, c_{1}, d_{2}, c_{2}\right)$ and $\mathfrak{a}_{2}\left(\mathfrak{a}_{1}\left(a_{1}, b_{2}\right.\right.$, $\left.\left.c_{1}\right), c_{2}, d_{2}\right) \in$ the carrier of $\operatorname{BitFTA} 1 \operatorname{Str}\left(a_{1}, b_{2}, c_{1}, d_{2}, c_{2}\right)$ and $\left\langle\left\langle\mathfrak{a}_{1}\left(a_{1}\right.\right.\right.$, $\left.\left.\left.b_{2}, c_{1}\right), c_{2}\right\rangle, \operatorname{and}_{2 a}\right\rangle \in$ the carrier of $\operatorname{BitFTA} 1 \operatorname{Str}\left(a_{1}, b_{2}, c_{1}, d_{2}, c_{2}\right)$ and $\left\langle\left\langle c_{2}, d_{2}\right\rangle\right.$, and2c $\rangle \in$ the carrier of $\operatorname{BitFTA} 1 \operatorname{Str}\left(a_{1}, b_{2}, c_{1}, d_{2}, c_{2}\right)$ and $\left\langle\left\langle d_{2}\right.\right.$, $\left.\mathfrak{a}_{1}\left(a_{1}, b_{2}, c_{1}\right)\right\rangle$, and $\left.{ }_{2 b}\right\rangle \in$ the carrier of $\operatorname{BitFTA} 1 \operatorname{Str}\left(a_{1}, b_{2}, c_{1}, d_{2}, c_{2}\right)$ and $\mathfrak{c}_{2}\left(\mathfrak{a}_{1}\left(a_{1}, b_{2}, c_{1}\right), c_{2}, d_{2}\right) \in$ the carrier of $\operatorname{BitFTA} 1 \operatorname{Str}\left(a_{1}, b_{2}, c_{1}, d_{2}, c_{2}\right)$.

(15) Let $a_{1}, b_{2}, c_{1}, d_{2}, c_{2}$ be sets. Then $\left\langle\left\langle a_{1}, b_{2}\right\rangle\right.$, xor2c $\rangle \in \mathcal{I} \mathcal{V}\left(\operatorname{BitFTA} 1 \operatorname{Str}\left(a_{1}\right.\right.$, $\left.\left.b_{2}, c_{1}, d_{2}, c_{2}\right)\right)$ and $\mathfrak{a}_{1}\left(a_{1}, b_{2}, c_{1}\right) \in \mathcal{I} \mathcal{V}\left(\operatorname{BitFTA} 1 \operatorname{Str}\left(a_{1}, b_{2}, c_{1}, d_{2}, c_{2}\right)\right)$ and $\left\langle\left\langle a_{1}, b_{2}\right\rangle\right.$, and2c $\rangle,\left\langle\left\langle b_{2}, c_{1}\right\rangle, \operatorname{and}_{2 a}\right\rangle,\left\langle\left\langle c_{1}, a_{1}\right\rangle, \operatorname{and}_{2}\right\rangle \in \mathcal{I V}\left(\operatorname{BitFTA} 1 \operatorname{Str}\left(a_{1}\right.\right.$, $\left.\left.b_{2}, c_{1}, d_{2}, c_{2}\right)\right)$ and $\mathfrak{c}_{1}\left(a_{1}, b_{2}, c_{1}\right) \in \mathcal{I V}\left(\operatorname{BitFTA} 1 \operatorname{Str}\left(a_{1}, b_{2}, c_{1}, d_{2}, c_{2}\right)\right)$ and $\left\langle\left\langle\mathfrak{a}_{1}\left(a_{1}, b_{2}, c_{1}\right), c_{2}\right\rangle\right.$, xor2c $\rangle, \mathfrak{a}_{2}\left(\mathfrak{a}_{1}\left(a_{1}, b_{2}, c_{1}\right), c_{2}, d_{2}\right),\left\langle\left\langle\mathfrak{a}_{1}\left(a_{1}, b_{2}, c_{1}\right), c_{2}\right\rangle\right.$, $\left.\operatorname{and}_{2 a}\right\rangle,\left\langle\left\langle c_{2}, d_{2}\right\rangle\right.$, and2c $\rangle,\left\langle\left\langle d_{2}, \mathfrak{a}_{1}\left(a_{1}, b_{2}, c_{1}\right)\right\rangle\right.$, and $\left.{ }_{2 b}\right\rangle, \mathfrak{c}_{2}\left(\mathfrak{a}_{1}\left(a_{1}, b_{2}, c_{1}\right)\right.$, $\left.c_{2}, d_{2}\right) \in \mathcal{I} \mathcal{V}\left(\operatorname{BitFTA} 1 \operatorname{Str}\left(a_{1}, b_{2}, c_{1}, d_{2}, c_{2}\right)\right)$.

(16) Let $a_{1}, b_{2}, c_{1}, d_{2}$ be non pair sets and $c_{2}$ be a set. Suppose $c_{2} \neq\left\langle\left\langle d_{2}\right.\right.$, $\left.\mathfrak{a}_{1}\left(a_{1}, b_{2}, c_{1}\right)\right\rangle$, $\left.\operatorname{and}_{2 b}\right\rangle$ and $c_{2} \notin \mathcal{I V}\left(\Sigma_{1}\left(a_{1}, b_{2}, c_{1}\right)\right)$. Then $a_{1}, b_{2}, c_{1}, d_{2}$, $c_{2} \in \operatorname{InputVertices}\left(\operatorname{BitFTA} 1 \operatorname{Str}\left(a_{1}, b_{2}, c_{1}, d_{2}, c_{2}\right)\right)$.

Let $a_{1}, b_{2}, c_{1}, d_{2}, c_{2}$ be sets. The functor BitFTA1CarryOutput $\left(a_{1}, b_{2}, c_{1}\right.$, $\left.d_{2}, c_{2}\right)$ yielding an element of $\mathcal{I} \mathcal{V}\left(\operatorname{BitFTA} 1 \operatorname{Str}\left(a_{1}, b_{2}, c_{1}, d_{2}, c_{2}\right)\right)$ is defined as follows:

(Def. 9) BitFTA1CarryOutput $\left(a_{1}, b_{2}, c_{1}, d_{2}, c_{2}\right)=\mathfrak{c}_{1}\left(a_{1}, b_{2}, c_{1}\right)$.

The functor $\operatorname{BitFTA1AdderOutputI}\left(a_{1}, b_{2}, c_{1}, d_{2}, c_{2}\right)$ yields an element of $\mathcal{I V}\left(\operatorname{BitFTA} 1 \operatorname{Str}\left(a_{1}, b_{2}, c_{1}, d_{2}, c_{2}\right)\right)$ and is defined by:

(Def. 10) BitFTA1AdderOutputI $\left(a_{1}, b_{2}, c_{1}, d_{2}, c_{2}\right)=\mathfrak{a}_{1}\left(a_{1}, b_{2}, c_{1}\right)$.

The functor $\operatorname{BitFTA1AdderOutputP}\left(a_{1}, b_{2}, c_{1}, d_{2}, c_{2}\right)$ yields an element of $\mathcal{I V}$ (BitFTA1Str $\left.\left(a_{1}, b_{2}, c_{1}, d_{2}, c_{2}\right)\right)$ and is defined as follows:

(Def. 11) BitFTA1AdderOutputP $\left(a_{1}, b_{2}, c_{1}, d_{2}, c_{2}\right)=\mathfrak{c}_{2}\left(\mathfrak{a}_{1}\left(a_{1}, b_{2}, c_{1}\right), c_{2}, d_{2}\right)$.

The functor BitFTA1AdderOutputQ $\left(a_{1}, b_{2}, c_{1}, d_{2}, c_{2}\right)$ yielding an element of $\mathcal{I V}\left(\operatorname{BitFTA} 1 \operatorname{Str}\left(a_{1}, b_{2}, c_{1}, d_{2}, c_{2}\right)\right)$ is defined as follows:

(Def. 12) BitFTA1AdderOutputQ $\left(a_{1}, b_{2}, c_{1}, d_{2}, c_{2}\right)=\mathfrak{a}_{2}\left(\mathfrak{a}_{1}\left(a_{1}, b_{2}, c_{1}\right), c_{2}, d_{2}\right)$.

The following four propositions are true:

(17) Let $a_{1}, b_{2}, c_{1}$ be non pair sets, $d_{2}, c_{2}$ be sets, $s$ be a state of $\operatorname{BitFTA1Circ}\left(a_{1}, b_{2}, c_{1}, d_{2}, c_{2}\right)$, and $a_{2}, a_{3}, a_{4}$ be elements of Boolean. Suppose $a_{2}=s\left(a_{1}\right)$ and $a_{3}=s\left(b_{2}\right)$ and $a_{4}=s\left(c_{1}\right)$. Then $($ Following $(s, 2))\left(\operatorname{BitFTA1CarryOutput}\left(a_{1}, b_{2}, c_{1}, d_{2}, c_{2}\right)\right)=a_{2} \wedge \neg a_{3} \vee$ $\neg a_{3} \wedge a_{4} \vee a_{4} \wedge a_{2}$ and (Following $\left.(s, 2)\right)$ (BitFTA1AdderOutputI $\left(a_{1}, b_{2}, c_{1}\right.$, $\left.\left.d_{2}, c_{2}\right)\right)=\neg\left(a_{2} \oplus \neg a_{3} \oplus a_{4}\right)$.

(18) Let $a_{1}, b_{2}, c_{1}, d_{2}$ be non pair sets and $c_{2}$ be a set. Suppose $c_{2} \neq\left\langle\left\langle d_{2}\right.\right.$, $\left.\mathfrak{a}_{1}\left(a_{1}, b_{2}, c_{1}\right)\right\rangle$, and $\left.\operatorname{and}_{2 b}\right\rangle$ and $c_{2} \notin \mathcal{I V}\left(\Sigma_{1}\left(a_{1}, b_{2}, c_{1}\right)\right)$. Let $s$ be a state of $\operatorname{BitFTA1Circ}\left(a_{1}, b_{2}, c_{1}, d_{2}, c_{2}\right)$ and $a_{2}, a_{3}, a_{4}, a_{5}, a_{6}$ be elements of 
Boolean. Suppose $a_{2}=s\left(a_{1}\right)$ and $a_{3}=s\left(b_{2}\right)$ and $a_{4}=s\left(c_{1}\right)$ and $a_{5}=s\left(d_{2}\right)$ and $a_{6}=s\left(c_{2}\right)$. Then $($ Following $(s, 2))\left(\mathfrak{a}_{1}\left(a_{1}, b_{2}, c_{1}\right)\right)=$ $\neg\left(a_{2} \oplus \neg a_{3} \oplus a_{4}\right)$ and (Following $\left.(s, 2)\right)\left(a_{1}\right)=a_{2}$ and (Following $\left.(s, 2)\right)\left(b_{2}\right)=$ $a_{3}$ and (Following $\left.(s, 2)\right)\left(c_{1}\right)=a_{4}$ and (Following $\left.(s, 2)\right)\left(d_{2}\right)=a_{5}$ and (Following $(s, 2))\left(c_{2}\right)=a_{6}$.

(19) Let $a_{1}, b_{2}, c_{1}, d_{2}$ be non pair sets and $c_{2}$ be a set. Suppose $c_{2} \neq\left\langle\left\langle d_{2}\right.\right.$, $\left.\mathfrak{a}_{1}\left(a_{1}, b_{2}, c_{1}\right)\right\rangle$, and $\left.\operatorname{and}_{2 b}\right\rangle$ and $c_{2} \notin \mathcal{I V}\left(\Sigma_{1}\left(a_{1}, b_{2}, c_{1}\right)\right)$. Let $s$ be a state of $\operatorname{BitFTA1Circ}\left(a_{1}, b_{2}, c_{1}, d_{2}, c_{2}\right)$ and $a_{2}, a_{3}, a_{4}, a_{5}, a_{6}$ be elements of Boolean. Suppose $a_{2}=s\left(a_{1}\right)$ and $a_{3}=s\left(b_{2}\right)$ and $a_{4}=s\left(c_{1}\right)$ and $a_{5}=s\left(d_{2}\right)$ and $a_{6}=s\left(c_{2}\right)$. Then (Following $\left.(s, 4)\right)$ (BitFTA1AdderOutputP $\left(a_{1}, b_{2}\right.$, $\left.\left.c_{1}, d_{2}, c_{2}\right)\right)=\neg\left(\left(a_{2} \oplus \neg a_{3} \oplus a_{4}\right) \wedge a_{6} \vee a_{6} \wedge \neg a_{5} \vee \neg a_{5} \wedge\left(a_{2} \oplus \neg a_{3} \oplus a_{4}\right)\right)$ and (Following $(s, 4))\left(\operatorname{BitFTA1AdderOutputQ}\left(a_{1}, b_{2}, c_{1}, d_{2}, c_{2}\right)\right)=a_{2} \oplus \neg a_{3} \oplus$ $a_{4} \oplus \neg a_{5} \oplus a_{6}$.

(20) Let $a_{1}, b_{2}, c_{1}, d_{2}$ be non pair sets and $c_{2}$ be a set. If $c_{2} \neq\left\langle\left\langle d_{2}, \mathfrak{a}_{1}\left(a_{1}\right.\right.\right.$, $\left.\left.b_{2}, c_{1}\right)\right\rangle$, $\left.\operatorname{and}_{2 b}\right\rangle$, then for every state $s$ of $\operatorname{BitFTA1Circ}\left(a_{1}, b_{2}, c_{1}, d_{2}, c_{2}\right)$ holds Following $(s, 4)$ is stable.

\section{Stability of 4-2 Binary Addition Circuit Cell (TYPE-2)}

Let $a_{7}, b_{1}, c_{3}, d_{1}, c_{2}$ be sets. The functor $\operatorname{BitFTA} 2 \operatorname{Str}\left(a_{7}, b_{1}, c_{3}, d_{1}, c_{2}\right)$ yielding an unsplit non void strict non empty many sorted signature with arity held in gates and Boolean denotation held in gates is defined by:

(Def. 13) $\operatorname{BitFTA2Str}\left(a_{7}, b_{1}, c_{3}, d_{1}, c_{2}\right)=\Sigma_{2}\left(a_{7}, b_{1}, c_{3}\right)+\cdot \Sigma_{1}\left(\mathfrak{a}_{2}\left(a_{7}, b_{1}, c_{3}\right), c_{2}\right.$, $\left.d_{1}\right)$.

Let $a_{7}, b_{1}, c_{3}, d_{1}, c_{2}$ be sets. The functor $\operatorname{BitFTA2\operatorname {Circ}}\left(a_{7}, b_{1}, c_{3}, d_{1}, c_{2}\right)$ yielding a strict Boolean circuit of $\operatorname{BitFTA} 2 \operatorname{Str}\left(a_{7}, b_{1}, c_{3}, d_{1}, c_{2}\right)$ with denotation held in gates is defined by:

(Def. 14) $\operatorname{BitFTA2Circ}\left(a_{7}, b_{1}, c_{3}, d_{1}, c_{2}\right)=\mathfrak{C}_{2}\left(a_{7}, b_{1}, c_{3}\right)+\cdot \mathfrak{C}_{1}\left(\mathfrak{a}_{2}\left(a_{7}, b_{1}, c_{3}\right), c_{2}\right.$, $\left.d_{1}\right)$.

Next we state several propositions:

(21) Let $a_{7}, b_{1}, c_{3}, d_{1}, c_{2}$ be sets. Then $\mathcal{I V}\left(\operatorname{BitFTA2} \operatorname{Str}\left(a_{7}, b_{1}, c_{3}, d_{1}\right.\right.$, $\left.\left.c_{2}\right)\right)=\left\{\left\langle\left\langle a_{7}, b_{1}\right\rangle, \operatorname{xor} 2 c\right\rangle, \mathfrak{a}_{2}\left(a_{7}, b_{1}, c_{3}\right)\right\} \cup\left\{\left\langle\left\langle a_{7}, b_{1}\right\rangle, \operatorname{and}_{2 a}\right\rangle,\left\langle\left\langle b_{1}, c_{3}\right\rangle\right.\right.$, and2c $\rangle,\left\langle\left\langle c_{3}, a_{7}\right\rangle\right.$, $\left.\left.\operatorname{and}_{2 b}\right\rangle, \mathfrak{c}_{2}\left(a_{7}, b_{1}, c_{3}\right)\right\} \cup\left\{\left\langle\left\langle\mathfrak{a}_{2}\left(a_{7}, b_{1}, c_{3}\right), c_{2}\right\rangle\right.\right.$, xor2c $\rangle, \mathfrak{a}_{1}$ $\left.\left(\mathfrak{a}_{2}\left(a_{7}, b_{1}, c_{3}\right), c_{2}, d_{1}\right)\right\} \cup\left\{\left\langle\left\langle\mathfrak{a}_{2}\left(a_{7}, b_{1}, c_{3}\right), c_{2}\right\rangle\right.\right.$, and2c $\rangle,\left\langle\left\langle c_{2}, d_{1}\right\rangle, \operatorname{and}_{2 a}\right\rangle$, $\left.\left\langle\left\langle d_{1}, \mathfrak{a}_{2}\left(a_{7}, b_{1}, c_{3}\right)\right\rangle, \operatorname{and}_{2}\right\rangle, \mathfrak{c}_{1}\left(\mathfrak{a}_{2}\left(a_{7}, b_{1}, c_{3}\right), c_{2}, d_{1}\right)\right\}$.

(22) For all sets $a_{7}, b_{1}, c_{3}, d_{1}, c_{2}$ holds $\mathcal{I V}\left(\operatorname{BitFTA2} \operatorname{Str}\left(a_{7}, b_{1}, c_{3}, d_{1}, c_{2}\right)\right)$ is a binary relation.

(23) For all non pair sets $a_{7}, b_{1}, c_{3}, d_{1}$ and for every set $c_{2}$ such that $c_{2} \neq\left\langle\left\langle d_{1}, \mathfrak{a}_{2}\left(a_{7}, b_{1}, c_{3}\right)\right\rangle, \operatorname{and}_{2}\right\rangle$ and $c_{2} \notin \mathcal{I V}\left(\Sigma_{2}\left(a_{7}, b_{1}, c_{3}\right)\right)$ holds InputVertices(BitFTA2Str $\left.\left(a_{7}, b_{1}, c_{3}, d_{1}, c_{2}\right)\right)=\left\{a_{7}, b_{1}, c_{3}, d_{1}, c_{2}\right\}$. 
(24) Let $a_{7}, b_{1}, c_{3}, d_{1}, c_{2}$ be sets. Then $a_{7} \in$ the carrier of $\operatorname{BitFTA2Str}\left(a_{7}\right.$, $\left.b_{1}, c_{3}, d_{1}, c_{2}\right)$ and $b_{1} \in$ the carrier of $\operatorname{BitFTA2} \operatorname{Str}\left(a_{7}, b_{1}, c_{3}, d_{1}, c_{2}\right)$ and $c_{3} \in$ the carrier of $\operatorname{BitFTA2Str}\left(a_{7}, b_{1}, c_{3}, d_{1}, c_{2}\right)$ and $d_{1} \in$ the carrier of $\operatorname{BitFTA2Str}\left(a_{7}, b_{1}, c_{3}, d_{1}, c_{2}\right)$ and $c_{2} \in$ the carrier of $\operatorname{BitFTA2Str}\left(a_{7}, b_{1}\right.$, $\left.c_{3}, d_{1}, c_{2}\right)$ and $\left\langle\left\langle a_{7}, b_{1}\right\rangle\right.$, xor2c $\rangle \in$ the carrier of $\operatorname{BitFTA} 2 \operatorname{Str}\left(a_{7}, b_{1}, c_{3}\right.$, $\left.d_{1}, c_{2}\right)$ and $\mathfrak{a}_{2}\left(a_{7}, b_{1}, c_{3}\right) \in$ the carrier of $\operatorname{BitFTA2} \operatorname{Str}\left(a_{7}, b_{1}, c_{3}, d_{1}, c_{2}\right)$ and $\left\langle\left\langle a_{7}, b_{1}\right\rangle, \operatorname{and}_{2 a}\right\rangle \in$ the carrier of $\operatorname{BitFTA} 2 \operatorname{Str}\left(a_{7}, b_{1}, c_{3}, d_{1}, c_{2}\right)$ and $\left\langle\left\langle b_{1}, c_{3}\right\rangle\right.$, and2c $\rangle \in$ the carrier of $\operatorname{BitFTA} 2 \operatorname{Str}\left(a_{7}, b_{1}, c_{3}, d_{1}, c_{2}\right)$ and $\left\langle\left\langle c_{3}\right.\right.$, $\left.\left.a_{7}\right\rangle, \operatorname{and}_{2 b}\right\rangle \in$ the carrier of $\operatorname{BitFTA} 2 \operatorname{Str}\left(a_{7}, b_{1}, c_{3}, d_{1}, c_{2}\right)$ and $\mathfrak{c}_{2}\left(a_{7}, b_{1}\right.$, $\left.c_{3}\right) \in$ the carrier of $\operatorname{BitFTA2Str}\left(a_{7}, b_{1}, c_{3}, d_{1}, c_{2}\right)$ and $\left\langle\left\langle\mathfrak{a}_{2}\left(a_{7}, b_{1}, c_{3}\right), c_{2}\right\rangle\right.$, xor2c $\rangle \in$ the carrier of $\operatorname{BitFTA2Str}\left(a_{7}, b_{1}, c_{3}, d_{1}, c_{2}\right)$ and $\mathfrak{a}_{1}\left(\mathfrak{a}_{2}\left(a_{7}, b_{1}\right.\right.$, $\left.\left.c_{3}\right), c_{2}, d_{1}\right) \in$ the carrier of $\operatorname{BitFTA} 2 \operatorname{Str}\left(a_{7}, b_{1}, c_{3}, d_{1}, c_{2}\right)$ and $\left\langle\left\langle\mathfrak{a}_{2}\left(a_{7}\right.\right.\right.$, $\left.\left.b_{1}, c_{3}\right), c_{2}\right\rangle$, and2c $\rangle \in$ the carrier of $\operatorname{BitFTA2Str}\left(a_{7}, b_{1}, c_{3}, d_{1}, c_{2}\right)$ and $\left\langle\left\langle c_{2}, d_{1}\right\rangle, \operatorname{and}_{2 a}\right\rangle \in$ the carrier of $\operatorname{BitFTA} 2 \operatorname{Str}\left(a_{7}, b_{1}, c_{3}, d_{1}, c_{2}\right)$ and $\left\langle\left\langle d_{1}\right.\right.$, $\left.\left.\mathfrak{a}_{2}\left(a_{7}, b_{1}, c_{3}\right)\right\rangle, \operatorname{and}_{2}\right\rangle \in$ the carrier of $\operatorname{BitFTA} 2 \operatorname{Str}\left(a_{7}, b_{1}, c_{3}, d_{1}, c_{2}\right)$ and $\mathfrak{c}_{1}\left(\mathfrak{a}_{2}\left(a_{7}, b_{1}, c_{3}\right), c_{2}, d_{1}\right) \in$ the carrier of $\operatorname{BitFTA} 2 \operatorname{Str}\left(a_{7}, b_{1}, c_{3}, d_{1}, c_{2}\right)$.

(25) Let $a_{7}, b_{1}, c_{3}, d_{1}, c_{2}$ be sets. Then $\left\langle\left\langle a_{7}, b_{1}\right\rangle\right.$, xor2c $\rangle \in \mathcal{I} \mathcal{V}\left(\operatorname{BitFTA} 2 \operatorname{Str}\left(a_{7}\right.\right.$, $\left.\left.b_{1}, c_{3}, d_{1}, c_{2}\right)\right)$ and $\mathfrak{a}_{2}\left(a_{7}, b_{1}, c_{3}\right) \in \mathcal{I} \mathcal{V}\left(\operatorname{BitFTA} 2 \operatorname{Str}\left(a_{7}, b_{1}, c_{3}, d_{1}, c_{2}\right)\right)$ and $\left\langle\left\langle a_{7}, b_{1}\right\rangle, \operatorname{and}_{2 a}\right\rangle,\left\langle\left\langle b_{1}, c_{3}\right\rangle\right.$, and $\left.2 c\right\rangle,\left\langle\left\langle c_{3}, a_{7}\right\rangle, \operatorname{and}_{2 b}\right\rangle \in \mathcal{I} \mathcal{V}\left(\operatorname{BitFTA2Str}\left(a_{7}\right.\right.$, $\left.\left.b_{1}, c_{3}, d_{1}, c_{2}\right)\right)$ and $\mathfrak{c}_{2}\left(a_{7}, b_{1}, c_{3}\right) \in \mathcal{I V}\left(\operatorname{BitFTA} 2 \operatorname{Str}\left(a_{7}, b_{1}, c_{3}, d_{1}, c_{2}\right)\right)$ and $\left\langle\left\langle\mathfrak{a}_{2}\left(a_{7}, b_{1}, c_{3}\right), c_{2}\right\rangle\right.$, xor2c $\rangle, \mathfrak{a}_{1}\left(\mathfrak{a}_{2}\left(a_{7}, b_{1}, c_{3}\right), c_{2}, d_{1}\right),\left\langle\left\langle\mathfrak{a}_{2}\left(a_{7}, b_{1}, c_{3}\right), c_{2}\right\rangle\right.$, and2c $\rangle,\left\langle\left\langle c_{2}, d_{1}\right\rangle, \operatorname{and}_{2 a}\right\rangle,\left\langle\left\langle d_{1}, \mathfrak{a}_{2}\left(a_{7}, b_{1}, c_{3}\right)\right\rangle\right.$, and $\left.{ }_{2}\right\rangle, \mathfrak{c}_{1}\left(\mathfrak{a}_{2}\left(a_{7}, b_{1}, c_{3}\right)\right.$, $\left.c_{2}, d_{1}\right) \in \mathcal{I} \mathcal{V}\left(\operatorname{BitFTA} 2 \operatorname{Str}\left(a_{7}, b_{1}, c_{3}, d_{1}, c_{2}\right)\right)$.

(26) Let $a_{7}, b_{1}, c_{3}, d_{1}$ be non pair sets and $c_{2}$ be a set. Suppose $c_{2} \neq\left\langle\left\langle d_{1}\right.\right.$, $\left.\mathfrak{a}_{2}\left(a_{7}, b_{1}, c_{3}\right)\right\rangle$, and $\left.d_{2}\right\rangle$ and $c_{2} \notin \mathcal{I V}\left(\Sigma_{2}\left(a_{7}, b_{1}, c_{3}\right)\right)$. Then $a_{7}, b_{1}, c_{3}, d_{1}$, $c_{2} \in$ InputVertices(BitFTA2Str $\left.\left(a_{7}, b_{1}, c_{3}, d_{1}, c_{2}\right)\right)$.

Let $a_{7}, b_{1}, c_{3}, d_{1}, c_{2}$ be sets. The functor BitFTA2CarryOutput $\left(a_{7}, b_{1}, c_{3}\right.$, $\left.d_{1}, c_{2}\right)$ yields an element of $\mathcal{I} \mathcal{V}\left(\operatorname{BitFTA} 2 \operatorname{Str}\left(a_{7}, b_{1}, c_{3}, d_{1}, c_{2}\right)\right)$ and is defined as follows:

(Def. 15) BitFTA2CarryOutput $\left(a_{7}, b_{1}, c_{3}, d_{1}, c_{2}\right)=\mathfrak{c}_{2}\left(a_{7}, b_{1}, c_{3}\right)$.

The functor BitFTA2AdderOutputI $\left(a_{7}, b_{1}, c_{3}, d_{1}, c_{2}\right)$ yields an element of $\mathcal{I V}\left(\operatorname{BitFTA} 2 \operatorname{Str}\left(a_{7}, b_{1}, c_{3}, d_{1}, c_{2}\right)\right)$ and is defined as follows:

(Def. 16) BitFTA2AdderOutputI $\left(a_{7}, b_{1}, c_{3}, d_{1}, c_{2}\right)=\mathfrak{a}_{2}\left(a_{7}, b_{1}, c_{3}\right)$.

The functor BitFTA2AdderOutputP $\left(a_{7}, b_{1}, c_{3}, d_{1}, c_{2}\right)$ yields an element of $\mathcal{I V}\left(\operatorname{BitFTA} 2 \operatorname{Str}\left(a_{7}, b_{1}, c_{3}, d_{1}, c_{2}\right)\right)$ and is defined by:

(Def. 17) BitFTA2AdderOutputP $\left(a_{7}, b_{1}, c_{3}, d_{1}, c_{2}\right)=\mathfrak{c}_{1}\left(\mathfrak{a}_{2}\left(a_{7}, b_{1}, c_{3}\right), c_{2}, d_{1}\right)$.

The functor BitFTA2AdderOutputQ $\left(a_{7}, b_{1}, c_{3}, d_{1}, c_{2}\right)$ yielding an element of $\mathcal{I V}\left(\operatorname{BitFTA} 2 \operatorname{Str}\left(a_{7}, b_{1}, c_{3}, d_{1}, c_{2}\right)\right)$ is defined as follows:

(Def. 18) BitFTA2AdderOutputQ $\left(a_{7}, b_{1}, c_{3}, d_{1}, c_{2}\right)=\mathfrak{a}_{1}\left(\mathfrak{a}_{2}\left(a_{7}, b_{1}, c_{3}\right), c_{2}, d_{1}\right)$.

One can prove the following propositions: 
(27) Let $a_{7}, b_{1}, c_{3}$ be non pair sets, $d_{1}, c_{2}$ be sets, $s$ be a state of $\operatorname{BitFTA2\operatorname {Circ}}\left(a_{7}, b_{1}, c_{3}, d_{1}, c_{2}\right)$, and $a_{2}, a_{3}, a_{4}$ be elements of Boolean. Suppose $a_{2}=s\left(a_{7}\right)$ and $a_{3}=s\left(b_{1}\right)$ and $a_{4}=s\left(c_{3}\right)$. Then (Following $(s, 2))\left(\operatorname{BitFTA2CarryOutput}\left(a_{7}, b_{1}, c_{3}, d_{1}, c_{2}\right)\right)=\neg\left(\neg a_{2} \wedge a_{3} \vee\right.$ $\left.a_{3} \wedge \neg a_{4} \vee \neg a_{4} \wedge \neg a_{2}\right)$ and (Following $\left.(s, 2)\right)$ (BitFTA2AdderOutputI $\left(a_{7}, b_{1}\right.$, $\left.\left.c_{3}, d_{1}, c_{2}\right)\right)=\neg a_{2} \oplus a_{3} \oplus \neg a_{4}$.

(28) Let $a_{7}, b_{1}, c_{3}, d_{1}$ be non pair sets and $c_{2}$ be a set. Suppose $c_{2} \neq\left\langle\left\langle d_{1}\right.\right.$, $\left.\mathfrak{a}_{2}\left(a_{7}, b_{1}, c_{3}\right)\right\rangle$, and $\left.{ }_{2}\right\rangle$ and $c_{2} \notin \mathcal{I V}\left(\Sigma_{2}\left(a_{7}, b_{1}, c_{3}\right)\right)$. Let $s$ be a state of $\operatorname{BitFTA2Circ}\left(a_{7}, b_{1}, c_{3}, d_{1}, c_{2}\right)$ and $a_{2}, a_{3}, a_{4}, a_{5}, a_{6}$ be elements of Boolean. Suppose $a_{2}=s\left(a_{7}\right)$ and $a_{3}=s\left(b_{1}\right)$ and $a_{4}=s\left(c_{3}\right)$ and $a_{5}=s\left(d_{1}\right)$ and $a_{6}=s\left(c_{2}\right)$. Then (Following $\left.(s, 2)\right)\left(\mathfrak{a}_{2}\left(a_{7}, b_{1}, c_{3}\right)\right)=$ $\neg a_{2} \oplus a_{3} \oplus \neg a_{4}$ and (Following $\left.(s, 2)\right)\left(a_{7}\right)=a_{2}$ and (Following $\left.(s, 2)\right)\left(b_{1}\right)=$ $a_{3}$ and (Following $\left.(s, 2)\right)\left(c_{3}\right)=a_{4}$ and (Following $\left.(s, 2)\right)\left(d_{1}\right)=a_{5}$ and (Following $(s, 2))\left(c_{2}\right)=a_{6}$.

(29) Let $a_{7}, b_{1}, c_{3}, d_{1}$ be non pair sets and $c_{2}$ be a set. Suppose $c_{2} \neq\left\langle\left\langle d_{1}\right.\right.$, $\left.\mathfrak{a}_{2}\left(a_{7}, b_{1}, c_{3}\right)\right\rangle$, and $\left.{ }_{2}\right\rangle$ and $c_{2} \notin \mathcal{I V}\left(\Sigma_{2}\left(a_{7}, b_{1}, c_{3}\right)\right)$. Let $s$ be a state of $\operatorname{BitFTA2Circ}\left(a_{7}, b_{1}, c_{3}, d_{1}, c_{2}\right)$ and $a_{2}, a_{3}, a_{4}, a_{5}, a_{6}$ be elements of Boolean. Suppose $a_{2}=s\left(a_{7}\right)$ and $a_{3}=s\left(b_{1}\right)$ and $a_{4}=s\left(c_{3}\right)$ and $a_{5}=s\left(d_{1}\right)$ and $a_{6}=s\left(c_{2}\right)$. Then (Following $(s, 4)$ )(BitFTA2AdderOutputP $\left(a_{7}, b_{1}\right.$, $\left.\left.c_{3}, d_{1}, c_{2}\right)\right)=\left(\neg a_{2} \oplus a_{3} \oplus \neg a_{4}\right) \wedge \neg a_{6} \vee \neg a_{6} \wedge a_{5} \vee a_{5} \wedge\left(\neg a_{2} \oplus a_{3} \oplus \neg a_{4}\right)$ and (Following $(s, 4))\left(\operatorname{BitFTA2AdderOutputQ}\left(a_{7}, b_{1}, c_{3}, d_{1}, c_{2}\right)\right)=\neg\left(\neg a_{2} \oplus\right.$ $\left.a_{3} \oplus \neg a_{4} \oplus a_{5} \oplus \neg a_{6}\right)$.

(30) Let $a_{7}, b_{1}, c_{3}, d_{1}$ be non pair sets and $c_{2}$ be a set. If $c_{2} \neq\left\langle\left\langle d_{1}, \mathfrak{a}_{2}\left(a_{7}, b_{1}\right.\right.\right.$, $\left.\left.c_{3}\right)\right\rangle$, and $\left.{ }_{2}\right\rangle$, then for every state $s$ of $\operatorname{BitFTA2} \operatorname{Circ}\left(a_{7}, b_{1}, c_{3}, d_{1}, c_{2}\right)$ holds Following $(s, 4)$ is stable.

\section{Stability of 4-2 Binary Addition Circuit Cell (TYPE-3)}

Let $a_{7}, b_{2}, c_{3}, d_{2}, c_{2}$ be sets. The functor $\operatorname{BitFTA} 3 \operatorname{Str}\left(a_{7}, b_{2}, c_{3}, d_{2}, c_{2}\right)$ yields an unsplit non void strict non empty many sorted signature with arity held in gates and Boolean denotation held in gates and is defined by:

(Def. 19) $\operatorname{BitFTA3Str}\left(a_{7}, b_{2}, c_{3}, d_{2}, c_{2}\right)=\Sigma_{3}\left(a_{7}, b_{2}, c_{3}\right)+\cdot \Sigma_{3}\left(\mathfrak{a}_{3}\left(a_{7}, b_{2}, c_{3}\right), c_{2}\right.$, $\left.d_{2}\right)$.

Let $a_{7}, b_{2}, c_{3}, d_{2}, c_{2}$ be sets. The functor $\operatorname{BitFTA3\operatorname {Circ}}\left(a_{7}, b_{2}, c_{3}, d_{2}, c_{2}\right)$ yielding a strict Boolean circuit of $\operatorname{BitFTA3Str}\left(a_{7}, b_{2}, c_{3}, d_{2}, c_{2}\right)$ with denotation held in gates is defined by:

(Def. 20) $\operatorname{BitFTA3Circ}\left(a_{7}, b_{2}, c_{3}, d_{2}, c_{2}\right)=\mathfrak{C}_{3}\left(a_{7}, b_{2}, c_{3}\right)+\cdot \mathfrak{C}_{3}\left(\mathfrak{a}_{3}\left(a_{7}, b_{2}, c_{3}\right), c_{2}\right.$, $\left.d_{2}\right)$.

We now state several propositions: 
(31) Let $a_{7}, b_{2}, c_{3}, d_{2}, c_{2}$ be sets. Then $\mathcal{I V}\left(\operatorname{BitFTA3Str}\left(a_{7}, b_{2}, c_{3}, d_{2}, c_{2}\right)\right)=$ $\left\{\left\langle\left\langle a_{7}, b_{2}\right\rangle\right.\right.$, xor $\left.\left._{2}\right\rangle, \mathfrak{a}_{3}\left(a_{7}, b_{2}, c_{3}\right)\right\} \cup\left\{\left\langle\left\langle a_{7}, b_{2}\right\rangle, \operatorname{and}_{2 b}\right\rangle,\left\langle\left\langle b_{2}, c_{3}\right\rangle, \operatorname{and}_{2 b}\right\rangle,\left\langle\left\langle c_{3}\right.\right.\right.$, $\left.\left.\left.a_{7}\right\rangle, \operatorname{and}_{2 b}\right\rangle, \mathfrak{c}_{3}\left(a_{7}, b_{2}, c_{3}\right)\right\} \cup\left\{\left\langle\left\langle\mathfrak{a}_{3}\left(a_{7}, b_{2}, c_{3}\right), c_{2}\right\rangle, \operatorname{xor}_{2}\right\rangle, \mathfrak{a}_{3}\left(\mathfrak{a}_{3}\left(a_{7}, b_{2}, c_{3}\right)\right.\right.$, $\left.\left.c_{2}, d_{2}\right)\right\} \cup\left\{\left\langle\left\langle\mathfrak{a}_{3}\left(a_{7}, b_{2}, c_{3}\right), c_{2}\right\rangle, \operatorname{and}_{2 b}\right\rangle,\left\langle\left\langle c_{2}, d_{2}\right\rangle, \operatorname{and}_{2 b}\right\rangle,\left\langle\left\langle d_{2}, \mathfrak{a}_{3}\left(a_{7}, b_{2}, c_{3}\right)\right\rangle\right.\right.$, $\left.\left.\operatorname{and}_{2 b}\right\rangle, \mathfrak{c}_{3}\left(\mathfrak{a}_{3}\left(a_{7}, b_{2}, c_{3}\right), c_{2}, d_{2}\right)\right\}$.

(32) For all sets $a_{7}, b_{2}, c_{3}, d_{2}, c_{2}$ holds $\mathcal{I V}\left(\operatorname{BitFTA3Str}\left(a_{7}, b_{2}, c_{3}, d_{2}, c_{2}\right)\right)$ is a binary relation.

(33) For all non pair sets $a_{7}, b_{2}, c_{3}, d_{2}$ and for every set $c_{2}$ such that $c_{2} \neq\left\langle\left\langle d_{2}, \mathfrak{a}_{3}\left(a_{7}, b_{2}, c_{3}\right)\right\rangle\right.$, and $\left.\operatorname{an}_{2 b}\right\rangle$ and $c_{2} \notin \mathcal{I V}\left(\Sigma_{3}\left(a_{7}, b_{2}, c_{3}\right)\right)$ holds InputVertices(BitFTA3Str $\left.\left(a_{7}, b_{2}, c_{3}, d_{2}, c_{2}\right)\right)=\left\{a_{7}, b_{2}, c_{3}, d_{2}, c_{2}\right\}$.

(34) Let $a_{7}, b_{2}, c_{3}, d_{2}, c_{2}$ be sets. Then $a_{7} \in$ the carrier of $\operatorname{BitFTA3Str}\left(a_{7}\right.$, $\left.b_{2}, c_{3}, d_{2}, c_{2}\right)$ and $b_{2} \in$ the carrier of $\operatorname{BitFTA3Str}\left(a_{7}, b_{2}, c_{3}, d_{2}, c_{2}\right)$ and $c_{3} \in$ the carrier of $\operatorname{BitFTA3Str}\left(a_{7}, b_{2}, c_{3}, d_{2}, c_{2}\right)$ and $d_{2} \in$ the carrier of $\operatorname{BitFTA3Str}\left(a_{7}, b_{2}, c_{3}, d_{2}, c_{2}\right)$ and $c_{2} \in$ the carrier of $\operatorname{BitFTA3Str}\left(a_{7}\right.$, $\left.b_{2}, c_{3}, d_{2}, c_{2}\right)$ and $\left\langle\left\langle a_{7}, b_{2}\right\rangle\right.$, xor $\left._{2}\right\rangle \in$ the carrier of $\operatorname{BitFTA3Str}\left(a_{7}, b_{2}, c_{3}\right.$, $\left.d_{2}, c_{2}\right)$ and $\mathfrak{a}_{3}\left(a_{7}, b_{2}, c_{3}\right) \in$ the carrier of $\operatorname{BitFTA3Str}\left(a_{7}, b_{2}, c_{3}, d_{2}, c_{2}\right)$ and $\left\langle\left\langle a_{7}, b_{2}\right\rangle, \operatorname{and}_{2 b}\right\rangle \in$ the carrier of $\operatorname{BitFTA3Str}\left(a_{7}, b_{2}, c_{3}, d_{2}, c_{2}\right)$ and $\left\langle\left\langle b_{2}, c_{3}\right\rangle, \operatorname{and}_{2 b}\right\rangle \in$ the carrier of $\operatorname{BitFTA3Str}\left(a_{7}, b_{2}, c_{3}, d_{2}, c_{2}\right)$ and $\left\langle\left\langle c_{3}\right.\right.$, $\left.\left.a_{7}\right\rangle, \operatorname{and}_{2 b}\right\rangle \in$ the carrier of $\operatorname{BitFTA3Str}\left(a_{7}, b_{2}, c_{3}, d_{2}, c_{2}\right)$ and $\mathfrak{c}_{3}\left(a_{7}, b_{2}\right.$, $\left.c_{3}\right) \in$ the carrier of $\operatorname{BitFTA} 3 \operatorname{Str}\left(a_{7}, b_{2}, c_{3}, d_{2}, c_{2}\right)$ and $\left\langle\left\langle\mathfrak{a}_{3}\left(a_{7}, b_{2}, c_{3}\right), c_{2}\right\rangle\right.$, xor $\left._{2}\right\rangle \in$ the carrier of $\operatorname{BitFTA3Str}\left(a_{7}, b_{2}, c_{3}, d_{2}, c_{2}\right)$ and $\mathfrak{a}_{3}\left(\mathfrak{a}_{3}\left(a_{7}, b_{2}, c_{3}\right)\right.$, $\left.c_{2}, d_{2}\right) \in$ the carrier of $\operatorname{BitFTA3Str}\left(a_{7}, b_{2}, c_{3}, d_{2}, c_{2}\right)$ and $\left\langle\left\langle\mathfrak{a}_{3}\left(a_{7}, b_{2}, c_{3}\right)\right.\right.$, $\left.\left.c_{2}\right\rangle, \operatorname{and}_{2 b}\right\rangle \in$ the carrier of $\operatorname{BitFTA3Str}\left(a_{7}, b_{2}, c_{3}, d_{2}, c_{2}\right)$ and $\left\langle\left\langle c_{2}, d_{2}\right\rangle\right.$, $\left.\operatorname{and}_{2 b}\right\rangle \in$ the carrier of $\operatorname{BitFTA3Str}\left(a_{7}, b_{2}, c_{3}, d_{2}, c_{2}\right)$ and $\left\langle\left\langle d_{2}, \mathfrak{a}_{3}\left(a_{7}, b_{2}\right.\right.\right.$, $\left.\left.c_{3}\right)\right\rangle$, and $\left.\operatorname{an}_{2 b}\right\rangle \in$ the carrier of $\operatorname{BitFTA3Str}\left(a_{7}, b_{2}, c_{3}, d_{2}, c_{2}\right)$ and $\mathfrak{c}_{3}\left(\mathfrak{a}_{3}\left(a_{7}\right.\right.$, $\left.\left.b_{2}, c_{3}\right), c_{2}, d_{2}\right) \in$ the carrier of $\operatorname{BitFTA} 3 \operatorname{Str}\left(a_{7}, b_{2}, c_{3}, d_{2}, c_{2}\right)$.

(35) Let $a_{7}, b_{2}, c_{3}, d_{2}, c_{2}$ be sets. Then $\left\langle\left\langle a_{7}, b_{2}\right\rangle, \operatorname{xor}_{2}\right\rangle \in \mathcal{I V}\left(\operatorname{BitFTA3Str}\left(a_{7}\right.\right.$, $\left.\left.b_{2}, c_{3}, d_{2}, c_{2}\right)\right)$ and $\mathfrak{a}_{3}\left(a_{7}, b_{2}, c_{3}\right) \in \mathcal{I V}\left(\operatorname{BitFTA3Str}\left(a_{7}, b_{2}, c_{3}, d_{2}, c_{2}\right)\right)$ and $\left\langle\left\langle a_{7}, b_{2}\right\rangle, \operatorname{and}_{2 b}\right\rangle,\left\langle\left\langle b_{2}, c_{3}\right\rangle, \operatorname{and}_{2 b}\right\rangle,\left\langle\left\langle c_{3}, a_{7}\right\rangle, \operatorname{and}_{2 b}\right\rangle \in \mathcal{I V}\left(\operatorname{BitFTA3Str}\left(a_{7}\right.\right.$, $\left.\left.b_{2}, c_{3}, d_{2}, c_{2}\right)\right)$ and $\mathfrak{c}_{3}\left(a_{7}, b_{2}, c_{3}\right) \in \mathcal{I V}\left(\operatorname{BitFTA3Str}\left(a_{7}, b_{2}, c_{3}, d_{2}, c_{2}\right)\right)$ and $\left\langle\left\langle\mathfrak{a}_{3}\left(a_{7}, b_{2}, c_{3}\right), c_{2}\right\rangle, \operatorname{xor}_{2}\right\rangle, \mathfrak{a}_{3}\left(\mathfrak{a}_{3}\left(a_{7}, b_{2}, c_{3}\right), c_{2}, d_{2}\right),\left\langle\left\langle\mathfrak{a}_{3}\left(a_{7}, b_{2}, c_{3}\right)\right.\right.$, $\left.\left.c_{2}\right\rangle, \operatorname{and}_{2 b}\right\rangle,\left\langle\left\langle c_{2}, d_{2}\right\rangle, \operatorname{and}_{2 b}\right\rangle,\left\langle\left\langle d_{2}, \mathfrak{a}_{3}\left(a_{7}, b_{2}, c_{3}\right)\right\rangle, \operatorname{and}_{2 b}\right\rangle, \mathfrak{c}_{3}\left(\mathfrak{a}_{3}\left(a_{7}, b_{2}\right.\right.$, $\left.\left.c_{3}\right), c_{2}, d_{2}\right) \in \mathcal{I} \mathcal{V}\left(\operatorname{BitFTA} 3 \operatorname{Str}\left(a_{7}, b_{2}, c_{3}, d_{2}, c_{2}\right)\right)$.

(36) Let $a_{7}, b_{2}, c_{3}, d_{2}$ be non pair sets and $c_{2}$ be a set. Suppose $c_{2} \neq\left\langle\left\langle d_{2}\right.\right.$, $\left.\mathfrak{a}_{3}\left(a_{7}, b_{2}, c_{3}\right)\right\rangle$, and $\left.\operatorname{lib}_{2 b}\right\rangle$ and $c_{2} \notin \mathcal{I V}\left(\Sigma_{3}\left(a_{7}, b_{2}, c_{3}\right)\right)$. Then $a_{7}, b_{2}, c_{3}, d_{2}$, $c_{2} \in$ InputVertices(BitFTA3Str $\left.\left(a_{7}, b_{2}, c_{3}, d_{2}, c_{2}\right)\right)$.

Let $a_{7}, b_{2}, c_{3}, d_{2}, c_{2}$ be sets. The functor BitFTA3CarryOutput $\left(a_{7}, b_{2}, c_{3}\right.$, $\left.d_{2}, c_{2}\right)$ yields an element of $\mathcal{I} \mathcal{V}\left(\operatorname{BitFTA} \operatorname{Str}\left(a_{7}, b_{2}, c_{3}, d_{2}, c_{2}\right)\right)$ and is defined by:

(Def. 21) BitFTA3CarryOutput $\left(a_{7}, b_{2}, c_{3}, d_{2}, c_{2}\right)=\mathfrak{c}_{3}\left(a_{7}, b_{2}, c_{3}\right)$.

The functor $\operatorname{BitFTA3AdderOutputI}\left(a_{7}, b_{2}, c_{3}, d_{2}, c_{2}\right)$ yields an element of 
$\mathcal{I V}\left(\operatorname{BitFTA3Str}\left(a_{7}, b_{2}, c_{3}, d_{2}, c_{2}\right)\right)$ and is defined by:

(Def. 22) BitFTA3AdderOutputI $\left(a_{7}, b_{2}, c_{3}, d_{2}, c_{2}\right)=\mathfrak{a}_{3}\left(a_{7}, b_{2}, c_{3}\right)$.

The functor BitFTA3AdderOutputP $\left(a_{7}, b_{2}, c_{3}, d_{2}, c_{2}\right)$ yields an element of $\mathcal{I V}\left(\operatorname{BitFTA} \operatorname{Str}\left(a_{7}, b_{2}, c_{3}, d_{2}, c_{2}\right)\right)$ and is defined by:

(Def. 23) BitFTA3AdderOutputP $\left(a_{7}, b_{2}, c_{3}, d_{2}, c_{2}\right)=\mathfrak{c}_{3}\left(\mathfrak{a}_{3}\left(a_{7}, b_{2}, c_{3}\right), c_{2}, d_{2}\right)$.

The functor BitFTA3AdderOutputQ $\left(a_{7}, b_{2}, c_{3}, d_{2}, c_{2}\right)$ yielding an element of $\mathcal{I V}\left(\operatorname{BitFTA} 3 \operatorname{Str}\left(a_{7}, b_{2}, c_{3}, d_{2}, c_{2}\right)\right)$ is defined by:

(Def. 24) BitFTA3AdderOutputQ $\left(a_{7}, b_{2}, c_{3}, d_{2}, c_{2}\right)=\mathfrak{a}_{3}\left(\mathfrak{a}_{3}\left(a_{7}, b_{2}, c_{3}\right), c_{2}, d_{2}\right)$.

One can prove the following propositions:

(37) Let $a_{7}, b_{2}, c_{3}$ be non pair sets, $d_{2}, c_{2}$ be sets, $s$ be a state of $\operatorname{BitFTA3Circ}\left(a_{7}, b_{2}, c_{3}, d_{2}, c_{2}\right)$, and $a_{2}, a_{3}, a_{4}$ be elements of Boolean. Suppose $a_{2}=s\left(a_{7}\right)$ and $a_{3}=s\left(b_{2}\right)$ and $a_{4}=s\left(c_{3}\right)$. Then (Following $\left.(s, 2)\right)$ (BitFTA3CarryOutput $\left(a_{7}\right.$, $\left.\left.b_{2}, c_{3}, d_{2}, c_{2}\right)\right)=\neg\left(\neg a_{2} \wedge \neg a_{3} \vee \neg a_{3} \wedge \neg a_{4} \vee \neg a_{4} \wedge \neg a_{2}\right)$ and $($ Following $(s, 2))\left(\operatorname{BitFTA3AdderOutputI}\left(a_{7}, b_{2}, c_{3}, d_{2}, c_{2}\right)\right)=\neg\left(\neg a_{2} \oplus\right.$ $\left.\neg a_{3} \oplus \neg a_{4}\right)$.

(38) Let $a_{7}, b_{2}, c_{3}, d_{2}$ be non pair sets and $c_{2}$ be a set. Suppose $c_{2} \neq\left\langle\left\langle d_{2}\right.\right.$, $\left.\mathfrak{a}_{3}\left(a_{7}, b_{2}, c_{3}\right)\right\rangle$, and $\left.{ }_{2 b}\right\rangle$ and $c_{2} \notin \mathcal{I V}\left(\Sigma_{3}\left(a_{7}, b_{2}, c_{3}\right)\right)$. Let $s$ be a state of $\operatorname{BitFTA3\operatorname {Circ}}\left(a_{7}, b_{2}, c_{3}, d_{2}, c_{2}\right)$ and $a_{2}, a_{3}, a_{4}, a_{5}, a_{6}$ be elements of Boolean. Suppose $a_{2}=s\left(a_{7}\right)$ and $a_{3}=s\left(b_{2}\right)$ and $a_{4}=s\left(c_{3}\right)$ and $a_{5}=$ $s\left(d_{2}\right)$ and $a_{6}=s\left(c_{2}\right)$. Then (Following $\left.(s, 2)\right)\left(\mathfrak{a}_{3}\left(a_{7}, b_{2}, c_{3}\right)\right)=\neg\left(\neg a_{2} \oplus\right.$ $\left.\neg a_{3} \oplus \neg a_{4}\right)$ and (Following $\left.(s, 2)\right)\left(a_{7}\right)=a_{2}$ and (Following $\left.(s, 2)\right)\left(b_{2}\right)=$ $a_{3}$ and (Following $\left.(s, 2)\right)\left(c_{3}\right)=a_{4}$ and (Following $\left.(s, 2)\right)\left(d_{2}\right)=a_{5}$ and (Following $(s, 2))\left(c_{2}\right)=a_{6}$.

(39) Let $a_{7}, b_{2}, c_{3}, d_{2}$ be non pair sets and $c_{2}$ be a set. Suppose $c_{2} \neq\left\langle\left\langle d_{2}\right.\right.$, $\left.\mathfrak{a}_{3}\left(a_{7}, b_{2}, c_{3}\right)\right\rangle$, and $\left.\operatorname{an}_{2 b}\right\rangle$ and $c_{2} \notin \mathcal{I V}\left(\Sigma_{3}\left(a_{7}, b_{2}, c_{3}\right)\right)$. Let $s$ be a state of $\operatorname{BitFTA3Circ}\left(a_{7}, b_{2}, c_{3}, d_{2}, c_{2}\right)$ and $a_{2}, a_{3}, a_{4}, a_{5}, a_{6}$ be elements of Boolean. Suppose $a_{2}=s\left(a_{7}\right)$ and $a_{3}=s\left(b_{2}\right)$ and $a_{4}=s\left(c_{3}\right)$ and $a_{5}=s\left(d_{2}\right)$ and $a_{6}=s\left(c_{2}\right)$. Then (Following $\left.(s, 4)\right)$ (BitFTA3AdderOutputP $\left(a_{7}, b_{2}\right.$, $\left.\left.c_{3}, d_{2}, c_{2}\right)\right)=\neg\left(\left(\neg a_{2} \oplus \neg a_{3} \oplus \neg a_{4}\right) \wedge \neg a_{6} \vee \neg a_{6} \wedge \neg a_{5} \vee \neg a_{5} \wedge\left(\neg a_{2} \oplus\right.\right.$ $\left.\neg a_{3} \oplus \neg a_{4}\right)$ ) and (Following $\left.(s, 4)\right)$ (BitFTA3AdderOutputQ $\left(a_{7}, b_{2}, c_{3}, d_{2}\right.$, $\left.\left.c_{2}\right)\right)=\neg\left(\neg a_{2} \oplus \neg a_{3} \oplus \neg a_{4} \oplus \neg a_{5} \oplus \neg a_{6}\right)$.

(40) Let $a_{7}, b_{2}, c_{3}, d_{2}$ be non pair sets and $c_{2}$ be a set. If $c_{2} \neq\left\langle\left\langle d_{2}, a_{3}\left(a_{7}\right.\right.\right.$, $\left.\left.b_{2}, c_{3}\right)\right\rangle$, and $\left.a_{2 b}\right\rangle$, then for every state $s$ of $\operatorname{BitFTA3Circ}\left(a_{7}, b_{2}, c_{3}, d_{2}, c_{2}\right)$ holds Following $(s, 4)$ is stable.

\section{REFERENCES}

[1] Grzegorz Bancerek. König's theorem. Formalized Mathematics, 1(3):589-593, 1990.

[2] Grzegorz Bancerek and Yatsuka Nakamura. Full adder circuit. Part I. Formalized Mathematics, 5(3):367-380, 1996. 
[3] Czesław Byliński. Functions and their basic properties. Formalized Mathematics, 1(1):5565, 1990.

[4] Yatsuka Nakamura and Grzegorz Bancerek. Combining of circuits. Formalized Mathematics, 5(2):283-295, 1996.

[5] Yatsuka Nakamura, Piotr Rudnicki, Andrzej Trybulec, and Pauline N. Kawamoto. Introduction to circuits, II. Formalized Mathematics, 5(2):273-278, 1996.

[6] Yatsuka Nakamura, Piotr Rudnicki, Andrzej Trybulec, and Pauline N. Kawamoto. Preliminaries to circuits, II. Formalized Mathematics, 5(2):215-220, 1996.

[7] Takaya Nishiyama and Yasuho Mizuhara. Binary arithmetics. Formalized Mathematics, 4(1):83-86, 1993.

[8] Andrzej Trybulec. Enumerated sets. Formalized Mathematics, 1(1):25-34, 1990.

[9] Andrzej Trybulec. Many sorted algebras. Formalized Mathematics, 5(1):37-42, 1996.

[10] Zinaida Trybulec. Properties of subsets. Formalized Mathematics, 1(1):67-71, 1990.

[11] E. Jean Vuillemin. A very fast multiplication algorithm for VLSI implementation, integration. The VLSI Journal, 1(1):39-52, 1983.

[12] Katsumi Wasaki and Pauline N. Kawamoto. 2's complement circuit. Formalized Mathematics, 6(2):189-197, 1997.

[13] Edmund Woronowicz. Many-argument relations. Formalized Mathematics, 1(4):733-737, 1990.

[14] Edmund Woronowicz. Relations and their basic properties. Formalized Mathematics, 1(1):73-83, 1990.

[15] Shin'nosuke Yamaguchi, Katsumi Wasaki, and Nobuhiro Shimoi. Generalized full adder circuits (GFAs). Part I. Formalized Mathematics, 13(4):549-571, 2005.

Received August 28, 2008 